\title{
BMJ Open Health care utilisation following childhood acute lymphoblastic leukaemia: a population-based matched cohort study
}

\author{
Karen Schow Jensen (D) , ${ }^{1}$ Birgitte Klug Albertsen, ${ }^{1}$ Henrik Schrøder, ${ }^{1}$ \\ Alina Zalounina Falborg, ${ }^{2}$ Kjeld Schmiegelow, ${ }^{3,4}$ Steen Rosthøj, ${ }^{5}$ \\ Michael Thude Callesen, ${ }^{6}$ Peter Vedsted ${ }^{2}$
}

To cite: Jensen KS, Klug Albertsen B, Schrøder H, et al. Health care utilisation following childhood acute lymphoblastic leukaemia: a population-based matched cohort study. BMJ Open 2021;11:e049847. doi:10.1136/ bmjopen-2021-049847

- Prepublication history and additional supplemental material for this paper are available online. To view these files, please visit the journal online (http://dx.doi.org/10.1136/ bmjopen-2021-049847).

Received 04 February 2021 Accepted 13 October 2021

Check for updates

(C) Author(s) (or their employer(s)) 2021. Re-use permitted under CC BY-NC. No commercial re-use. See rights and permissions. Published by BMJ.

For numbered affiliations see end of article.

Correspondence to Karen Schow Jensen; kascje@rm.dk

\section{ABSTRACT}

Objectives Survival among children diagnosed with acute lymphoblastic leukaemia (ALL) has increased considerably. However, morbidity in survivors constitutes a potential increasing burden not limited to secondary health care. Our objectives were to compare health care utilisation, including both primary and secondary health care, between childhood ALL survivors and matched references up to 15 years after curative treatment. Moreover, to increase knowledge on survivors' health service seeking behaviour as time from treatment elapsed.

Design and setting A Danish population-based matched cohort study linking multiple nationwide registries.

Participants 675 cases, diagnosed with childhood (1.0-17.9 years) ALL between 1994 and 2015, and 6750 matched references sampled randomly from the source population (matched on age, gender and geographical region).

Primary outcome measures Repeated consultations in general practice and hospital (outpatient and inpatient) estimated as yearly rates from 2.5 years after diagnosis and onwards. We compared cases and references with yearly incidence rate ratios (IRRs) from negative binomial regression models.

Results Survivors of childhood ALL had a mean number of yearly daytime contacts in general practice of 4.75 (95\% Cl 4.41 to 5.11$)$ the first year, corresponding to an IRR of 1.85 (95\% Cl 1.71 to 2.00); decreasing to 1.16 (1.01 to 1.34 ) after 15 years, and without significant impact of gender $(p=0.894)$ or age $(p=0.399)$. For hospital contacts, ALL survivors had a mean number of yearly contacts of 14.21 (13.38-15.08) the first year, corresponding to an IRR of 31.50 (28.29-35.07); decreasing to 2.42 (1.59-3.68) after 15 years. No differences were found across calendar time.

Conclusions ALL survivors used significantly more health care services across sectors than the reference population. Decreasing use over 15 years illustrated the dynamics of health care needs; this knowledge may inform the future organisation of integrated follow-up programmes. Trial registration number NCT03985826.

\section{STRENGTHS AND LIMITATIONS OF THIS STUDY}

$\Rightarrow$ This is the first study to assess use of general practice in a population of childhood acute lymphoblastic leukaemia survivors, and not a broad spectrum of childhood cancer survivors.

$\Rightarrow$ The longitudinal data with measuring of repeated events provide valuable insight into long-term changes of health care utilisation as time from treatment elapsed.

$\Rightarrow$ Use of complete nationwide registries with nearly no loss to follow-up linked on an individual level ensures that the study was population-based and limits selection bias.

$\Rightarrow$ A large, matched population-based reference group provides a high statistical precision.

$\Rightarrow$ Information on all possible confounders was not available; however, due to a high number of matched references and the random identification, residual confounding is minimised.

\section{INTRODUCTION}

Acute lymphoblastic leukaemia (ALL) is the most frequent childhood cancer. In recent decades, advances in ALL treatment have increased the 5-year survival rate to above $90 \% .^{12}$ Recent studies have demonstrated that long-term survivors of childhood cancer, including ALL, have an increased risk of hospitalisations $^{3-11}$ and have more chronic conditions than references. ${ }^{12-15}$

The majority of previous studies including non-hospital-based contacts with doctors found survivors of childhood cancer to have increased utilisation of general practice services, ${ }^{10}$ 16-19 with one study presenting similar use of general practice in survivors of childhood cancer and references 11 years after diagnosis. ${ }^{20}$ Participants in these studies had a diverse spectrum of childhood cancers. In some studies, data were self-reported thus increasing the risk of recall bias, and some 
studies had a short follow-up time. ${ }^{1016-21}$ Studies on health care utilisation in general practice as a function of time since diagnosis are inconsistent; however, the majority of existing studies did not investigate this association. An inverse association between time since diagnosis and number of yearly visits was observed for the first 5 years after diagnosis. ${ }^{16}{ }^{17}$ McBride $e t ~ a l^{19}$ described no association between time since diagnosis and health care utilisation in later time periods. None of these studies reported results about changes in health care use in general practice across calendar time.

Thus, questions remain about the longitudinal excess health care utilisation including general practice as well as inpatient and outpatient hospital contacts, which may be used as proxy measures for health care needs and overall disease burden in this patient group. The aim of this study was to compare health care utilisation over time between ALL survivors and matched references, and to analyse if calendar time or treatment regime affected health care utilisation.

\section{METHODS}

\section{Study design and setting}

We conducted a nationwide, population-based matched cohort study using information from several Danish registries. Data on health care utilisation were extracted retrospectively for the period 1 January 1997 to 31 December 2018. Since 1997, all Danish children have been registered with their own unique Civil Personal Registration (CPR) number, enabling complete follow-up of each individual child. Before 1997, children's contacts to general practice were registered under their parents' CPR number. ${ }^{22}$ In Denmark, health care is primarily tax financed and free of charge for all citizens (population 5.8 million). The CPR number follows all citizens from birth to death, and data retrieved from Danish public registries were linked at individual level using the CPR number.

General practitioners act as gatekeepers in the Danish healthcare system except for emergencies and approximately $98 \%$ of the Danish population is registered with a specific general practice. ${ }^{23}$

We used the REporting of studies Conducted using Observational Routinely collected health Data statement checklist $^{24}$ as a guide for reporting of this study (online supplemental table $\mathrm{S} 1$ ).

\section{Participants}

Cases were defined as children (1.0-17.9 years) diagnosed with ALL between 1994 and 2015, and surviving at least 2.5 years after diagnosis (figure 1). Cases were identified in the Nordic Society of Paediatric Haematology and Oncology (NOPHO) ALL registry, which holds comprehensive data on all children aged 1.0-14.9 years in Denmark diagnosed with ALL since 1992. From 2008 and onwards, the ALL registry was extended to include children and adolescents aged $1.0-17.9$ years.

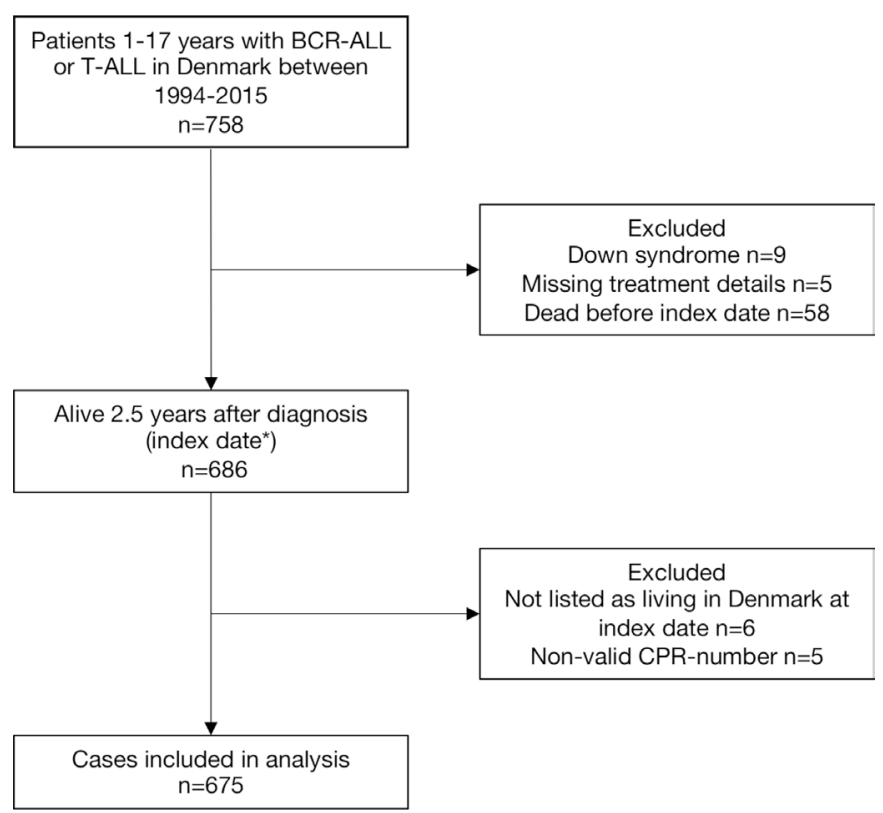

Figure 1 Flow diagram of the study population. ${ }^{*}$ Index date: 2.5 years after diagnosis of acute lymphoblastic leukaemia. BCR-ALL, B-cell precursor acute lymphoblastic leukaemia; CPR-number, Civil Personal Registration number; T-ALL, Tlineage acute lymphoblastic leukaemia.

The reference cohort was sampled randomly from the source population using the Danish Civil Registration System ${ }^{25}$ and matched by age, sex and geographical region among the five Danish regions and with no history of childhood cancer 2.5 years after the case was diagnosed (incidence density sampling). To check for history of childhood cancer, the reference cohort was checked in the Danish Cancer Registry, ${ }^{26}$ which holds information on all new cases of cancer in Denmark.

Each patient with ALL was matched with 10 reference subjects. Because of the population-based design, the study size was determined by the number of cases in the area during the study period. No sample size calculation was made.

\section{Data sources and variables}

Data from national population-based health registries were linked and hosted by Statistics Denmark. Postlinkage data were pseudoanonymised before the authors obtained access to the data.

We collected data on the following variables from the NOPHO registry: ALL treatment protocol (1992, 2000 or 2008), immunophenotype, haematopoietic stem cell transplantation and relapse. From the NOPHO registry, we further collected data on risk groups (non-high risk or high risk); at diagnosis, patients were stratified as high risk or non-high risk based on a number of characteristics: age, white blood cell count, involvement of extramedullary sites, immunophenotype, cytogenetics and response to treatment. A detailed description of risk group stratification according to the different treatment protocols has previously been published. ${ }^{127} 28$ We had no patient level information on treatment with cranial irradiation; 
however, in the NOPHO ALL1992 and NOPHO ALL2000 eras, the very high-risk group was treated with cranial irradiation according to protocol. ${ }^{27}$ Data on very high-risk group were obtained as a proxy for cranial irradiation. Information about sex, age, vital status, immigration and emigration was obtained from the Danish Civil Registration System. ${ }^{25}$ Information about the development of a second malignant neoplasm (SMN) was obtained from the Danish Cancer Registry. ${ }^{26}$

\section{Outcomes}

The primary outcomes were repeated contacts to general practice and hospital for up to 15 years after the end of curative ALL treatment. Data from general practice were obtained from the National Health Insurance Service Register. ${ }^{22}$ General practice data included contacts (daytime face-to-face contacts, out-of-hours contacts, email consultations, telephone consultations and home visits) and diagnostic procedures (blood test, urine test, streptococcus throat test, pulmonary functions test and ECG). We chose to include diagnostic procedures to provide a picture of the diagnostic activity level. We did not include preventive health examinations of children, vaccinations, screening for cervical cancer and pregnancy care. For a complete list of codes, see online supplemental table S2. Data from secondary health care were obtained from the Danish National Patient Registry. ${ }^{29}$ Secondary health care data included inpatient hospital care and outpatient visits at both public and private hospitals. Contacts to the accident and emergency department were excluded from the analyses as we expected no difference in visits between ALL survivors and matched references.

\section{Statistical methods}

Follow-up on health care contacts started 2.5 years after diagnosis for cases (the index date, which approximates the time of ending maintenance therapy) and on the corresponding day for the reference group. Study follow-up ended on the date of death, date of emigration or end of study (31 December 2018 for contacts related to general practice and 31 December 2017 for hospital contacts), whichever came first.

To estimate and compare yearly rates of health care contacts for cases and references, we used a negative binominal regression model. To account for differences in follow-up time, log-transformed risk time was included in the model with the regression parameter restricted to one. Cluster robust variance was applied to allow for intragroup correlation because repeated measurements (contacts) within individuals cannot be interpreted as independent events. Yearly rates for primary health care contacts (daytime contacts, out-of-hours contacts and diagnostic procedures) and secondary health care contacts (including inpatient hospitalisations and hospital outpatient contacts) were calculated from the index date and forward. Yearly contact rates were calculated as crude estimates.
When comparing the yearly rates of health care contacts for cases and references, we calculated an incidence rate ratio (IRR). IRRs were adjusted for sex and age on the index date. To adjust for age, we used restricted cubic splines with six knots to allow for a non-linear relationship. We conducted stratified analyses on sex and age group ( $<10$ years, $\geq 10$ years at index date). Wald tests were performed to test for interaction between the effect of ALL treatment with age group and sex across all yearly periods of follow-up.

Finally, analyses of ALL cases only were conducted comparing the two risk groups (the non-high-risk group was set as reference), the three calendar times according to treatment protocols (the NOPHO ALL1992 protocol was set as reference) and haematopoietic stem cell transplantation in first complete remission (no transplantation was set as reference).

We performed a sensitivity analysis to investigate the robustness of our results by excluding all cases undergoing haematopoietic stem cell transplantation at any time point. Moreover, we conducted a sensitivity analysis censoring all cases developing a relapse or an SMN on the date of the relapse/SMN diagnosis.

All estimates are presented with 95\% CIs. All tests were two sided and a $p$ value $<0.05$ was considered statistically significant. Data were analysed using the statistical software package Stata V.16.1 (StataCorp LLC, Texas, USA).

\section{Patient and public involvement}

The study included no patient and public involvement.

\section{RESULTS}

The study included 675 childhood ALL survivors and 6750 matched references with a total follow-up time of 81611 person-years and a median follow-up time of 11 years. Table 1 shows characteristics of the population. The overall 5-year survival rate was $97.5 \%$ (95\% CI 95.9 to 98.5) for the included 675 childhood ALL survivors (risk time starts at index date).

\section{Health care utilisation}

ALL survivors had a mean number of yearly daytime contacts in general practice of 4.75 (95\% CI 4.41 to 5.11) the first year after the index date, corresponding to an IRR of 1.85 (95\% CI 1.71 to 2.00); this declined to rates not statistically different from references during a 10-year period, IRR=1.11 (95\% CI 0.99 to 1.25$) 10$ years after the index date; however, the IRRs remained above one throughout the follow-up (figure 2). For out-of-hours contacts and diagnostic procedures, the IRRs comparing cases and references the first year after the index date were 2.14 (95\% CI 1.81 to 2.53 ) and 2.38 (95\% CI 2.05 to 2.77), respectively, (figure 2).

For hospital health care utilisation, ALL survivors had a mean number of yearly contacts of 14.21 (95\% CI 13.38 to 15.08), corresponding to an IRR of 31.50 (95\% CI 28.29 to 35.07 ) the first year after the index date, declining to 
Table 1 Characteristics of the study population

\begin{tabular}{|c|c|c|}
\hline Characteristics & $\begin{array}{l}\text { Cases } \\
\mathrm{N}=675\end{array}$ & $\begin{array}{l}\text { References } \\
\mathrm{N}=6750\end{array}$ \\
\hline \multicolumn{3}{|l|}{ Sex, n (\%) } \\
\hline Male & $379(56.1)$ & $3790(56.1)$ \\
\hline Female & $296(43.9)$ & $2960(43.9)$ \\
\hline Median age at index date (IQI) & $6.9(5.4-10.4)$ & $6.9(5.4-10.4)$ \\
\hline \multicolumn{3}{|l|}{ Age group at index date, $\mathrm{n}(\%)$} \\
\hline Age $<10$ years & $494(73.2)$ & $4940(73.2)$ \\
\hline Age $\geq 10$ years & $181(26.8)$ & $1810(26.8)$ \\
\hline \multicolumn{3}{|l|}{ Cell line, n (\%) } \\
\hline B-precursor ALL & $587(87.0)$ & - \\
\hline T-ALL & $88(13.0)$ & - \\
\hline \multicolumn{3}{|l|}{ Treatment protocol, $\mathrm{n}(\%)$} \\
\hline NOPHO ALL1992 & $231(34.2)$ & - \\
\hline NOPHO ALL2000 & $210(31.1)$ & - \\
\hline NOPHO ALL2008 & $234(34.7)$ & - \\
\hline \multicolumn{3}{|l|}{ Risk group, n (\%) } \\
\hline Non-high risk & $515(76.3)$ & - \\
\hline High risk/VHR* & $124 / 36(23.7)$ & - \\
\hline $\begin{array}{l}\text { Treated with HSCT in CR1, } n \\
(\%)\end{array}$ & $41(6.1)$ & - \\
\hline $\begin{array}{l}\text { Treated with HSCT at any point, } \\
\mathrm{n}(\%)\end{array}$ & $74(11.0)$ & - \\
\hline Relapse, n (\%) & $63(9.3)$ & - \\
\hline SMN, n (\%) & $14(2.1)$ & - \\
\hline $\begin{array}{l}\text { Median years of follow-up from } \\
\text { index date, (IQI) }\end{array}$ & $10.5(5.2-15.8)$ & $11.1(5.6-16.0)$ \\
\hline \multicolumn{3}{|c|}{$\begin{array}{l}675 \text { cases (childhood survivors of acute lymphoblastic leukaemia } \\
\text { (ALL)) and } 6750 \text { population-based references matched on age, sex } \\
\text { and geographical region on index date ( } 2.5 \text { years after diagnosis of } \\
\text { ALL for cases and the corresponding date for references). } \\
\text { *VHR: very high risk as a proxy for cranial irradiation. } \\
\text { HSCT in CR1, haematopoietic stem cell transplantation in first } \\
\text { complete remission; IQI, interquartile interval; NOPHO, Nordic Society } \\
\text { of Paediatric Haematology and Oncology; SMN, second malignant } \\
\text { neoplasm. }\end{array}$} \\
\hline
\end{tabular}

an IRR of 2.42 (95\% CI 1.59 to 3.68$) 15$ years after the index date (figure 3 ).

Comparing the three protocols (corresponding three calendar times) for ALL cases only revealed similar contact rates (figure 4). Comparing the two risk groups showed statistically significantly more contacts in the high-risk group, year 1 after the index date in general practice (figure 5). Comparing patients with ALL treated with or without haematopoietic stem cell transplantation (no transplantation was set as reference) revealed more contacts in the transplantation group (online supplemental figure S1).

Analyses stratified on age group and sex revealed no independent risk factors for use of health care after ALL treatment (online supplemental figures S2 and S3). This was supported by insignificant results from the tests for interaction between the effect of ALL treatment with sex
( $\mathrm{p}=0.894$ for general practice, $\mathrm{p}=0.067$ for hospital) and age $(\mathrm{p}=0.399$ for general practice, $\mathrm{p}=0.079$ for hospital)

\section{Sensitivity analyses}

In the analysis excluding all cases undergoing haematopoietic stem cell transplantation at any time point before or after index date, the overall findings remained the same (online supplemental figure S4). In the analysis, censoring all cases developing a relapse or an SMN on the date of relapse/SMN diagnosis did not change the overall findings (online supplemental figure S5).

\section{DISCUSSION}

The present population-based study was designed to investigate the longitudinal overall excess health care utilisation in survivors of childhood ALL from treatment cessation and onwards. Our results showed that ALL survivors had a higher utilisation of general practice and hospital health care services than their matched references, with a declining pattern over the 15-year period as time from treatment elapsed. For hospital health care utilisation, the number of yearly contacts showed a steep decline during the first 5 years. This finding is in agreement with survivors attending frequent but declining hospital-based follow-up during the first 5 years. ${ }^{30}$ It is noteworthy that the use of general practice services was high in the same time period as survivors attending frequent hospital-based follow-up.

No differences in health care utilisation emerged across calendar time (measured by treatment protocols). This has, to the best of our knowledge, not previously been analysed in a primary health care setting. As expected, we found increased health care use in patients following haematopoietic stem cell transplantation. ${ }^{31} 32$ Patients treated with cranial irradiation are included in the high-risk group, and we expected patients treated with cranial irradiation to have increased health care use. $^{41215202133-35}$ However, the increased utilisation in the high-risk group was only significant in year 1 in general practice. This could reflect that the number of patients exposed to cranial irradiation is low. Analyses stratified on age group and sex revealed no independent risk factors for use of health care after ALL treatment. As expected from previous research, ${ }^{36}$ we found an overall tendency towards increased use of general practice services in females compared with males. The differences found in ALL survivors corresponded to sex differences in the general population.

\section{Strengths and limitations}

The population-based design using nationwide registries linked on an individual level is a strength. The use of complete nationwide registries with nearly no loss to follow-up limits selection bias. Use of registry data on health care contacts covering a long period provides a picture of the temporal development in health care utilisation as time from treatment elapsed. Furthermore, 

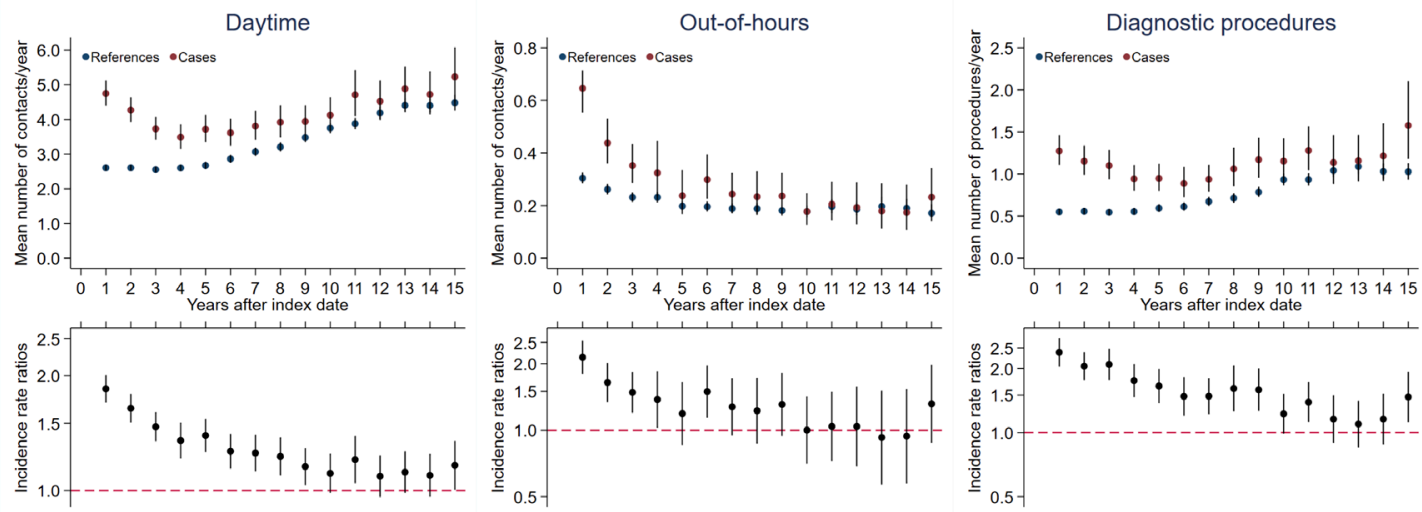

Figure 2 General practice health care utilisation during 15 years

.A total of 675 cases compared with 6750 references. Health care utilisation after the index date is divided into daytime consultations, out-of-hours consultations and diagnostic procedures in general practice. Top panel: Mean yearly number of contacts/diagnostic procedures presented as crude rates. Bottom panel: Incidence rate ratios adjusted for age and sex. Vertical lines represent $95 \%$ Cls. Index date: 2.5 years after diagnosis of acute lymphoblastic leukaemia for cases and the corresponding date for references.

a large matched population-based reference group is an advantage as it provides a high statistical precision. Data on exposure are robust to misclassification because cancer registration is mandatory in Denmark. ${ }^{26}$
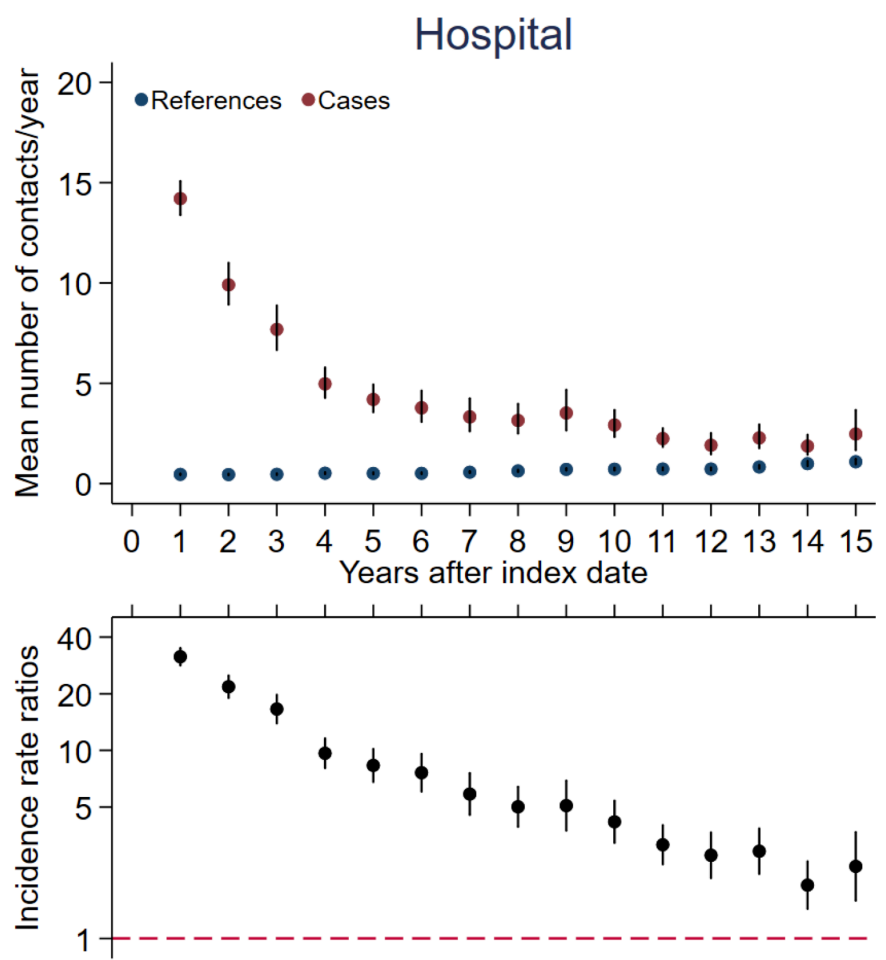

Figure 3 Hospital health care utilisation during 15 years. Hospital health care utilisation after the index date; 675 cases compared with 6750 references. Top panel: Mean yearly number of contacts presented as crude rates. Bottom panel: Incidence rate ratios adjusted for age and sex. Vertical lines represent $95 \%$ Cls.

Hospital health care utilisation: Including hospital inpatient and hospital outpatient contacts. Index date: 2.5 years after diagnosis of acute lymphoblastic leukaemia for cases and the corresponding date for references.
Health care data are collected routinely and uniformly in the Danish healthcare system. They are collected for all individuals and not for the purpose of the present study. We have no reason to believe that ALL cases had more or less complete registration of health care data, thereby minimising potential misclassification. ${ }^{37}$

The study period began 2.5 years after diagnosis to ensure that curative treatment had ended, which ensures a fairly homogeneous study group. However, we expect some cases to have extended treatment, explaining some of the increased rates of hospital contacts in the first year after the index date.

We reduced confounding by age, gender and geographical region by matching cases with references. Still, residual confounding will exist due to ethnicity and socioeconomic factors, including rural/urban status. However, due to the high number of matched references and the random identification, residual confounding may have been further minimised. We believe that our findings can be generalised to other countries with comparable healthcare systems.

\section{Comparison with other studies}

Previous studies on primary health care utilisation in childhood cancer survivors have been based on participants with a broad spectrum of childhood cancers and have investigated shorter periods either right after diagnosis or later in life. Two studies ${ }^{16}{ }^{17}$ investigated health care utilisation in the 5-year period from diagnosis. Both studies found increased use of general practice services among childhood cancer survivors, which is in agreement with our results. Shaw et $a t^{20}$ found similar use of general practice services among childhood cancer survivors and general population controls. This is not in agreement with our results; however, results are based on self-reported outcomes. Three studies ${ }^{10} 1819$ found an increased use of general practice in the survivor group compared with a comparison group later in life, including patients analysed up to 62 years after a cancer diagnosis. Data 
General practice
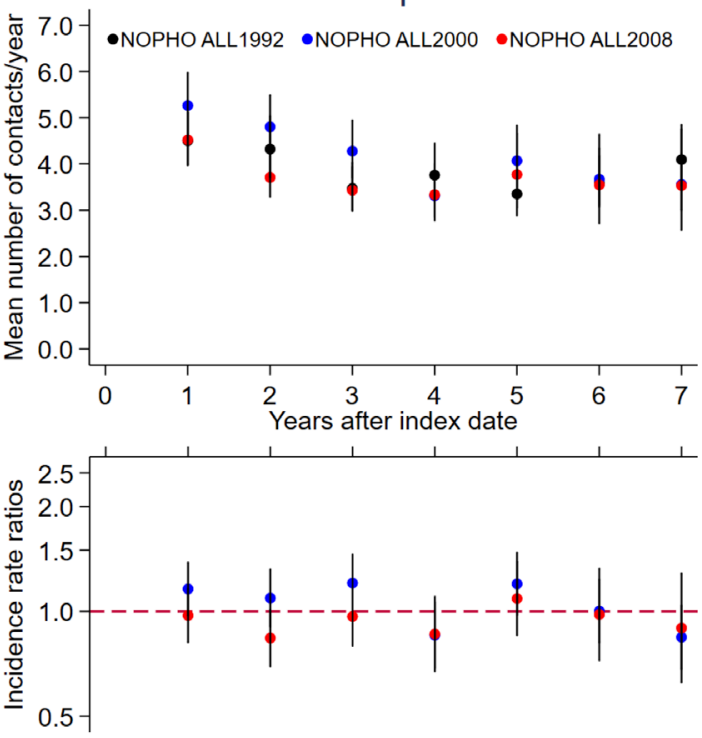

Hospital
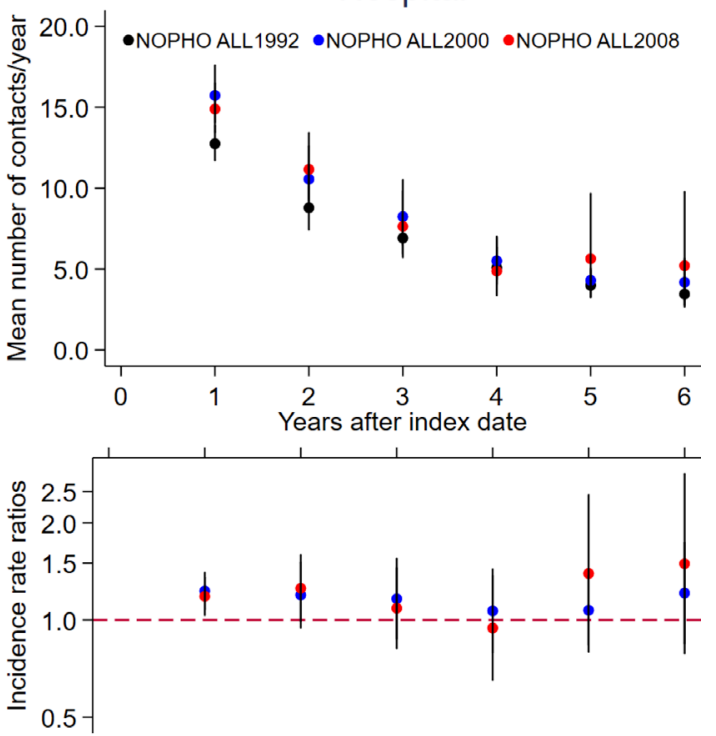

Figure 4 Health care utilisation comparing three calendar times.

cases only, health care utilisation by year after the index date comparing three calendar times (the NOPHO ALL treatment protocols from $1992(n=231), 2000(n=210)$ and $2008(n=234))$. Health care utilisation is stratified on health care setting (hospital and general practice). Top panel: Mean yearly number of contacts presented as crude rates. Bottom panel: Incidence rate ratios of ALL2000 and ALL2008 compared with ALL1992 (the ALL1992 protocol was set as reference) adjusted for age and sex. Vertical lines represent $95 \%$ Cls. Index date: 2.5 years after diagnosis of acute lymphoblastic leukaemia. NOPHO, the Nordic Society of Paediatric Haematology and Oncology.

in these studies are not presented with contact rate ratios as a function of time since diagnosis and they are thus difficult to compare with our results. In none of the mentioned studies, it was possible to compare different calendar times.
We studied both inpatient and outpatient hospital contacts, and calculated the contact rate ratio for each year following the end of ALL treatment. Two studies ${ }^{35}$ based on hospital admissions found that the hospitalisation rate
General practice
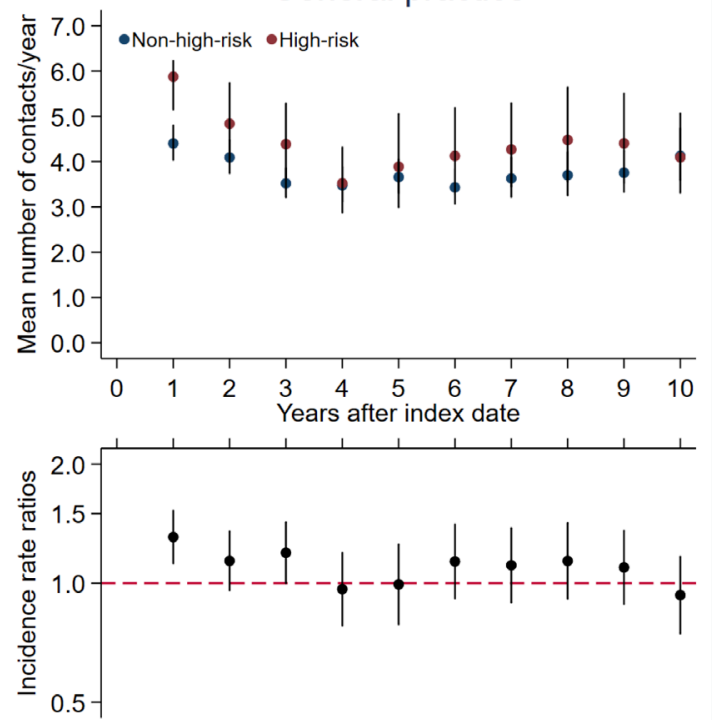

Hospital
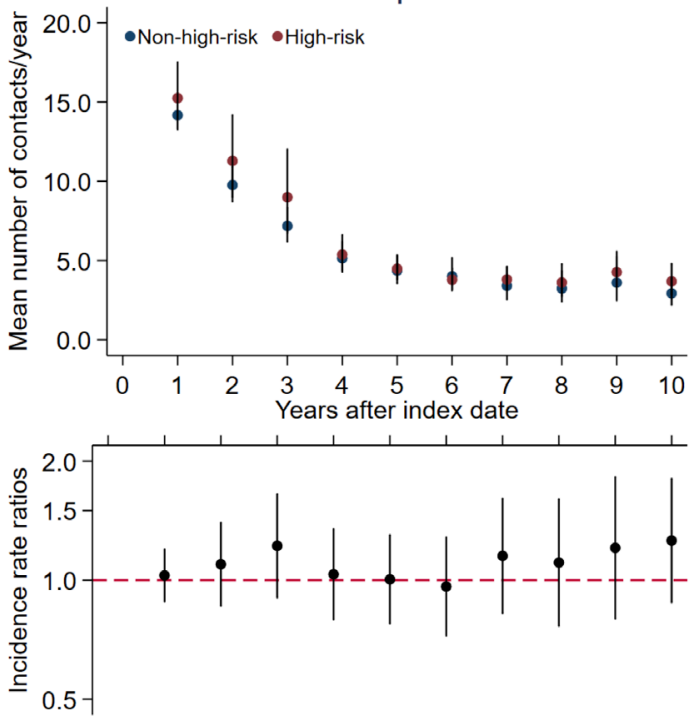

Figure 5 Health care utilisation comparing non-high-risk and high-risk groups.

ALL cases only, health care utilisation by year after the index date comparing non-high-risk $(n=515)$ and high-risk groups $(n=160)$ (ie, therapy). Health care utilisation is stratified on health care setting (hospital and general practice). Top panel: Mean yearly number of contacts presented as crude rates. Bottom panel: Incidence rate ratios of high risk compared with non-high risk as reference adjusted for age and sex. Vertical lines represent $95 \%$ Cls. Index date: 2.5 years after diagnosis of acute lymphoblastic leukaemia. 
ratio for leukaemia survivors was approximately two times higher than their references. This was in agreement with our results, at 15 years from index date. These studies presented no information about treatment regimen.

\section{CONCLUSIONS}

Our findings demonstrate that ALL survivors use health care in general practice, out-of-hours services and hospitals considerably more often than their peers. This indicates a continuing need for medical care across sectors for 10 or more years after treatment has ended. Thus, ALL survivors seem to have a high need for integrated and coordinated follow-up in general practice and hospitals; this knowledge may inform the organisation of future follow-up programmes for this group.

\section{Author affiliations}

${ }^{1}$ Department of Paediatrics and Adolescent Medicine, Aarhus University Hospital, Aarhus, Denmark

${ }^{2}$ Research Centre for Cancer Diagnosis in Primary Care, Research Unit for General Practice, Department of Public Health, Aarhus University, Aarhus, Denmark ${ }^{3}$ Department of Paediatrics and Adolescent Medicine, Copenhagen University Hospital, Copenhagen, Denmark

${ }^{4}$ Institute of Clinical Medicine, Faculty of Health and Medical Sciences, University of Copenhagen, Copenhagen, Denmark

${ }^{5}$ Department of Paediatrics and Adolescent Medicine, Aalborg University Hospital, Aalborg, Denmark

${ }^{6}$ Hans Christian Andersen Children's Hospital, Odense University Hospital, Odense, Denmark

Acknowledgements The authors wish to thank Kaare Rud Flarup for his assistance with data management.

Contributors KSJ designed the study, analysed and interpreted data and wrote the manuscript. BKA interpreted data and edited the manuscript. HS designed the study and edited the manuscript. AZF analysed and interpreted data and edited the manuscript. KS interpreted data and edited the manuscript. SR interpreted data and edited the manuscript. MTC interpreted data and edited the manuscript. PV designed the study, interpreted data, edited the manuscript and acted as guarantor. All authors approved the final manuscript.

Funding This work was supported by a grant from the Danish Cancer Society (Kræftens Bekæmpelse). Grant number R124-A7831-15-S2. The funders had no role in the design and conduct of the study.

Competing interests BKA declares the following: sponsor for the investigator initiated NOR-GRASPALL 2016 study. KS declares the following: Speaker and/or Advisory Board Honoraria from Jazz Pharmaceuticals (2020) and Servier (2020); speaker fee from Amgen (2020) and Medscape (2020); Educational grant from Servier (2020).

Patient consent for publication Not applicable.

Ethics approval This study was approved by the Danish Data Protection Agency (ID 277). Medical ethical approval was not required according to Danish law.

Provenance and peer review Not commissioned; externally peer reviewed.

Data availability statement No data are available. Data sharing statement: According to the data agreement with the data provider, we are not allowed to share our data. Data are stored and maintained electronically at Statistics Denmark.

Supplemental material This content has been supplied by the author(s). It has not been vetted by BMJ Publishing Group Limited (BMJ) and may not have been peer-reviewed. Any opinions or recommendations discussed are solely those of the author(s) and are not endorsed by BMJ. BMJ disclaims all liability and responsibility arising from any reliance placed on the content. Where the content includes any translated material, BMJ does not warrant the accuracy and reliability of the translations (including but not limited to local regulations, clinical guidelines, terminology, drug names and drug dosages), and is not responsible for any error and/or omissions arising from translation and adaptation or otherwise.
Open access This is an open access article distributed in accordance with the Creative Commons Attribution Non Commercial (CC BY-NC 4.0) license, which permits others to distribute, remix, adapt, build upon this work non-commercially, and license their derivative works on different terms, provided the original work is properly cited, appropriate credit is given, any changes made indicated, and the use is non-commercial. See: http://creativecommons.org/licenses/by-nc/4.0/.

ORCID iD

Karen Schow Jensen http://orcid.org/0000-0002-0811-8818

\section{REFERENCES}

1 Toft N, Birgens H, Abrahamsson J, et al. Results of NOPHO ALL2008 treatment for patients aged 1-45 years with acute lymphoblastic leukemia. Leukemia 2018;32:606-15.

2 Pui C-H, Campana D, Pei D, et al. Treating childhood acute lymphoblastic leukemia without cranial irradiation. $N$ Engl J Med 2009;360:2730-41

3 Sørensen GV, Winther JF, de Fine Licht S, et al. Long-Term risk of hospitalization among five-year survivors of childhood leukemia in the Nordic countries. J Nat/ Cancer Inst 2019;111:943-51.

4 Ou JY, Smits-Seemann RR, Kaul S, et al. Risk of hospitalization among survivors of childhood and adolescent acute lymphoblastic leukemia compared to siblings and a general population sample. Cancer Epidemiol 2017:49:216-24.

5 de Fine Licht S, Rugbjerg K, Gudmundsdottir T, et al. Long-Term inpatient disease burden in the adult life after childhood cancer in Scandinavia (ALiCCS) study: a cohort study of 21,297 childhood cancer survivors. PLoS Med 2017;14:e1002296.

6 Sieswerda E, Font-Gonzalez A, Reitsma JB, et al. High hospitalization rates in survivors of childhood cancer: a longitudinal follow-up study using medical record linkage. PLoS One 2016:11:e0159518.

7 Zhang Y, Lorenzi MF, Goddard K, et al. Late morbidity leading to hospitalization among 5-year survivors of young adult cancer: a report of the childhood, adolescent and young adult cancer survivors research program. Int J Cancer 2014;134:1174-82.

8 Lorenzi MF, Xie L, Rogers PC, et al. Hospital-related morbidity among childhood cancer survivors in British Columbia, Canada: report of the childhood, adolescent, young adult cancer survivors (CAYACS) program. Int J Cancer 2011;128:1624-31.

9 Kurt BA, Nolan VG, Ness KK, et al. Hospitalization rates among survivors of childhood cancer in the childhood cancer Survivor study cohort. Pediatr Blood Cancer 2012;59:126-32.

10 Rebholz CE, Reulen RC, Toogood AA, et al. Health care use of longterm survivors of childhood cancer: the British childhood cancer Survivor study. J Clin Oncol 2011;29:4181-8.

11 Font-Gonzalez A, Feijen ELAM, Geskus RB, et al. Risk and associated risk factors of hospitalization for specific health problems over time in childhood cancer survivors: a medical record linkage study. Cancer Med 2017;6:1123-34.

12 Mody R, Li S, Dover DC, et al. Twenty-Five-Year follow-up among survivors of childhood acute lymphoblastic leukemia: a report from the childhood cancer Survivor study. Blood 2008;111:5515-23.

13 Mulrooney DA, Hyun G, Ness KK, et al. The changing burden of longterm health outcomes in survivors of childhood acute lymphoblastic leukaemia: a retrospective analysis of the ST Jude lifetime cohort study. Lancet Haematol 2019;6:e306-16.

14 Oeffinger KC, Mertens AC, Sklar CA, et al. Chronic health conditions in adult survivors of childhood cancer. N Engl J Med 2006;355:1572-82.

15 Bhakta N, Liu Q, Ness KK, et al. The cumulative burden of surviving childhood cancer: an initial report from the ST Jude lifetime cohort study (SJLIFE). Lancet 2017;390:2569-82.

16 Heins MJ, Lorenzi MF, Korevaar JC, et al. Non-oncology physician visits after diagnosis of cancer in children. BMC Fam Pract 2016;17:60

17 Heins MJ, Lorenzi MF, Korevaar JC, et al. Non-oncology physician visits after diagnosis of cancer in adolescents and young adults. $J$ Cancer Surviv 2016;10:783-8.

18 Streefkerk N, Heins MJ, Teepen JC, et al. The involvement of primary care physicians in care for childhood cancer survivors. Pediatr Blood Cancer 2019;66:e27774.

19 McBride ML, Lorenzi MF, Page J, et al. Patterns of physician follow-up among young cancer survivors: report of the childhood, adolescent, and young adult cancer survivors (CAYACS) research program. Can Fam Physician 2011;57:e482-90.

20 Shaw AK, Pogany L, Speechley KN, et al. Use of health care services by survivors of childhood and adolescent cancer in Canada. Cancer 2006;106:1829-37. 
21 Oeffinger KC, Mertens AC, Hudson MM, et al. Health care of young adult survivors of childhood cancer: a report from the childhood cancer Survivor study. Ann Fam Med 2004;2:61-70.

22 Andersen JS, Olivarius NDF, Krasnik A. The Danish National health service register. Scand J Public Health 2011;39:34-7.

23 Pedersen KM, Andersen JS, Søndergaard J. General practice and primary health care in Denmark. J Am Board Fam Med 2012;25 Suppl 1:S34-8.

24 Benchimol El, Smeeth L, Guttmann A, et al. The reporting of studies conducted using observational Routinely-collected health data (record) statement. PLoS Med 2015;12:e1001885.

25 Schmidt M, Pedersen L, Sørensen HT. The Danish civil registration system as a tool in epidemiology. Eur J Epidemiol 2014;29:541-9.

26 Gjerstorff ML. The Danish cancer registry. Scand J Public Health 2011;39:42-5.

27 Schmiegelow K, Forestier E, Hellebostad M, et al. Long-Term results of NOPHO ALL-92 and ALL-2000 studies of childhood acute lymphoblastic leukemia. Leukemia 2010;24:345-54.

28 Gustafsson G, Schmiegelow K, Forestier E, et al. Improving outcome through two decades in childhood all in the Nordic countries: the impact of high-dose methotrexate in the reduction of CNS irradiation. Nordic Society of pediatric haematology and oncology (NOPHO). Leukemia 2000;14:2267-75.

29 Lynge E, Sandegaard JL, Rebolj M. The Danish national patient register. Scand J Public Health 2011;39:30-3.

30 Jensen KS, Oskarsson T, Lähteenmäki PM, et al. Detection mode of childhood acute lymphoblastic leukaemia relapse and its effect on survival: a Nordic population-based cohort study. Br J Haematol 2021;194:734-44.

31 Chow EJ, Anderson L, Baker KS, et al. Late effects surveillance recommendations among survivors of childhood hematopoietic cell transplantation: a children's Oncology Group report. Biol Blood Marrow Transplant 2016;22:782-95.

32 Armenian SH, Sun C-L, Kawashima T, et al. Long-Term health-related outcomes in survivors of childhood cancer treated with HSCT versus conventional therapy: a report from the bone marrow transplant Survivor study (BMTSS) and childhood cancer Survivor study (CCSS). Blood 2011;118:1413-20.

33 Holmqvist AS, Moëll C, Hjorth L, et al. Increased health care utilization by survivors of childhood lymphoblastic leukemia is confined to those treated with cranial or total body irradiation: a case cohort study. BMC Cancer 2014;14:419.

34 Spiegler BJ, Kennedy K, Maze R, et al. Comparison of long-term neurocognitive outcomes in young children with acute lymphoblastic leukemia treated with cranial radiation or high-dose or very highdose intravenous methotrexate. J Clin Oncol 2006;24:3858-64.

35 Dixon SB, Chen Y, Yasui Y, et al. Reduced morbidity and mortality in survivors of childhood acute lymphoblastic leukemia: a report from the childhood cancer Survivor study. J Clin Oncol 2020;38:3418-29.

36 Vedsted P. [Gender differences in the use of health care system]. Ugeskr Laeger 2007;169:2403-8.

37 Erlangsen A, Fedyszyn I. Danish nationwide registers for public health and health-related research. Scand J Public Health 2015;43:333-9. 